$1-1-1991$

\title{
Si(111)-(4×1)In surface reconstruction studied by impact-collision ion-scattering spectrometry
}

David M. Cornelison

M. S. Worthington

I. S.T. Tsong

Follow this and additional works at: https://bearworks.missouristate.edu/articles-cnas

\section{Recommended Citation}

Cornelison, D. M., M. S. Worthington, and I. S. T. Tsong. "Si (111)-(4× 1$)$ In surface reconstruction studied by impact-collision ion-scattering spectrometry." Physical Review B 43, no. 5 (1991): 4051.

This article or document was made available through BearWorks, the institutional repository of Missouri State University. The work contained in it may be protected by copyright and require permission of the copyright holder for reuse or redistribution.

For more information, please contact BearWorks@library.missouristate.edu. 


\title{
Si(111)-( $4 \times 1)$ In surface reconstruction studied by impact-collision ion-scattering spectrometry
}

\author{
D. M. Cornelison, M. S. Worthington, and I. S. T. Tsong \\ Department of Physics, Arizona State University, Tempe, Arizona 85287 \\ (Received 23 July 1990; revised manuscript received 24 October 1990)
}

\begin{abstract}
The technique of impact-collision ion-scattering spectrometry (ICISS) was used to study the $4 \times 1$ reconstruction of $\mathrm{In}$ on the $\mathrm{Si}(111)$ surface. The top layer of adatoms is arranged in double-row ridges, with three equivalent orientations running in $\langle\overline{1} 10\rangle$ directions. Several models have been simulated for both $\frac{1}{2}$-monolayer $(\mathrm{ML})$ and $1-\mathrm{ML}$ coverage of the surface. Our ICISS polar-angle scans do not agree with models containing substitutional In in the first Si layer. Instead, a $\frac{1}{2}-\mathrm{ML}$ model with In adatoms sitting in inequivalent sites provides the closest agreement with experiment. The vertical displacement between the In adatoms and the first Si layer has been determined experimentally to be $1.15 \pm 0.15 \AA$.
\end{abstract}

\section{INTRODUCTION}

The study of metal-induced reconstructions on semiconductor surfaces has been an active, important topic for many years. The initial growth stages of the groupIII metals on $\mathrm{Si}(111)$ quite often display several reconstructions for coverages from 0 to 1 monolayer (ML). Indium, specifically, induces at least three reconstructions, ${ }^{1}$ $\sqrt{3} \times \sqrt{3}$ at $\sim \frac{1}{3} \mathrm{ML}, \sqrt{31} \times \sqrt{31}$ at slightly higher coverage and, for something less than 1 -ML coverage, the $4 \times 1$ geometry. Of these three only the $\sqrt{3} \times \sqrt{3}$ phase has been studied extensively. ${ }^{2-4}$

The $4 \times 1$ reconstruction is not well understood. Twodimensional phase-diagram studies ${ }^{1}$ indicate the onset of the phase at $\frac{1}{2}-\mathrm{ML}$ coverage. Recent scanning tunneling microscopy (STM) results ${ }^{5}$ also show the top layer of the structure consists of ridges of double rows of atoms running along the $\langle\overline{1} 10\rangle$ directions, yielding three equivalent orientations. The exact positions of the adatoms are not completely clear from the STM images. ${ }^{5}$

In an earlier study ${ }^{6}$ by impact-collision ion-scattering spectrometry (ICISS), we performed preliminary work which suggested, based on a $\frac{1}{2}$-ML coverage, that the In adatoms most likely occupied threefold hollow $\left(\mathrm{H}_{3}\right)$ sites, as opposed to fourfold atop $\left(T_{4}\right)$ sites. These preliminary ICISS experiments were limited to polar-angle scans along only two major azimuths. In deriving the $\mathrm{H}_{3}$-site model, we ignored the possibility of a full monolayer coverage with substitutional second layer In. In the present paper, a number of different azimuths were scanned and the possibilities of second layer In and mixed $H_{3}$ and $T_{4}$ sites were also considered. By extending the study of the $4 \times 1$ reconstruction it is possible to compare results for $\frac{1}{2}-\mathrm{ML}$ and $1-\mathrm{ML}$ coverages and postulate models which are consistent with the experimental results. It is important to note that in the present work, the technique of ICISS is used to determine a viable model for a highly complex and poorly understood structure. This contrasts sharply with the usual practice of using ICISS to confirm already-existing models.

\section{EXPERIMENT}

The ICISS experiments were performed in an apparatus described previously. ${ }^{7}$ It consists of an ultrahigh-vacuum chamber equipped with reverse-view low-energy electron diffraction (LEED), two electrostatic energy analyzers, and a Colutron ion-beam system. The base pressure in the system was $\sim 1 \times 10^{-10}$ Torr. The $\mathrm{Si}(111)$ sample, cut from an $n$-type wafer of $0.02 \Omega \mathrm{cm}$ resistivity, was rinsed in ethanol and then mounted on a precision two-axis manipulator. The surface was annealed to produce a $7 \times 7$ geometry, which was confirmed using LEED. Indium was then deposited from a tungsten basket at a rate of $\sim 0.5 \mathrm{ML} / \mathrm{min}\left(1 \mathrm{ML} \approx 7.8 \times 10^{14}\right.$ $\mathrm{cm}^{-2} ; 1 \AA$ of $\left.\mathrm{In}=0.49 \mathrm{ML}\right)$. The deposition was monitored with a quartz crystal. After approximately $1 \mathrm{ML}$ of deposition, the surface was annealed at $350^{\circ} \mathrm{C}$ for $15-20$ $\min$. The duration of the anneal depended on the amount of In deposited, a longer annealing time was necessary for higher In coverages. LEED was then used to confirm the presence of the $4 \times 1$ reconstruction. In all cases, all three equivalent orientations, i.e., [ $\overline{1} 10],[10 \overline{1}]$, and [011 ], of the reconstruction existed simultaneously.

After preparation of the $4 \times 1$ surface, scattering experiments were conducted by bombarding the sample with a $2-\mathrm{keV} \mathrm{He}^{+}$-ion beam. The beam current was 30-50 nA and the spot size $4 \mathrm{~mm}$ in diameter. Polar-angle scans were run from $0^{\circ}$ to $60^{\circ}$ or $70^{\circ}$ in $2^{\circ}$ increments. Scattered ions were detected at either $1139 \mathrm{eV}$ (from Si) or $1745 \mathrm{eV}$ (from In) using an electrostatic energy analyzer set at a scattering angle of $163^{\circ}$. The time taken to complete a scan was approximately $5 \mathrm{~min}$, although the collection time at each angle was set longer for the Si scans.

\section{RESULTS AND DISCUSSION}

The starting point for a model of the $4 \times 1$ surface must begin with the STM results of Park, Nogami, and Quate. ${ }^{5}$ In the filled-state images of this reconstruction, it is clear that double rows of atoms on the surface run along equivalent $\langle\overline{1} 10\rangle$ directions. The perpendicular spacing 
between these double rows is $13.3 \AA$. The relative positions of the atoms within a double row shown in the STM images ${ }^{5}$ suggest an angle of $\sim 70^{\circ}$ between pairs of atoms within a double row and the axis of the double row (e.g., Figs. 1 and 2). Measurements of distances and angles from STM results are not, however, always accurate due to drifts and other factors. In our earlier simulations the In adatoms were put into equivalent sites (either $\mathrm{H}_{3}$ or $T_{4}$ ) even though this yields a $60^{\circ}$ angle between the double-row axis and the atom pairs in adjacent rows. We found that our experimental data favored the $H_{3}$ sites over the $T_{4}$ sites. A mixed-site geometry was not simulated and this could conceivably fit the experimental results. Substitutional In in the first Si layer was also not considered. The addition of In in the lower layers will certainly influence the simulated results.

Several models were considered in this paper. Two variations of a model proposed by Park, Nogami, and Quate $^{8}$ are termed models A and B. They are shown in Figs. 1 and 2, respectively. As stated previously, the $4 \times 1$ reconstruction exists in three equivalent $\langle\overline{1} 10\rangle$ directions. However, only one of these directions is shown in each of Figs. 1-5 for the sake of clarity. In both models $\mathrm{A}$ and $\mathrm{B}, \frac{1}{2} \mathrm{ML}$ of In lies in adatom positions on the top layer, forming the double rows. One adatom sits in an $\mathrm{H}_{3}$ site, one in a $T_{4}$ site. In model $\mathrm{A}$ (shown in Fig. 1) no substitutional In is present in the first Si layer. The total coverage is $\frac{1}{2} \mathrm{ML}$. For model $\mathrm{B}$, however, an additional $\frac{1}{2} \mathrm{ML}$ of $\mathrm{In}$ is substituted into the first Si layer giving a total In coverage of $1 \mathrm{ML}$. The Inadatom arrangement closely resembles the filled-state STM images.

Two models which locate the In adatoms in bridge sites were also considered. These are shown in Figs. 3

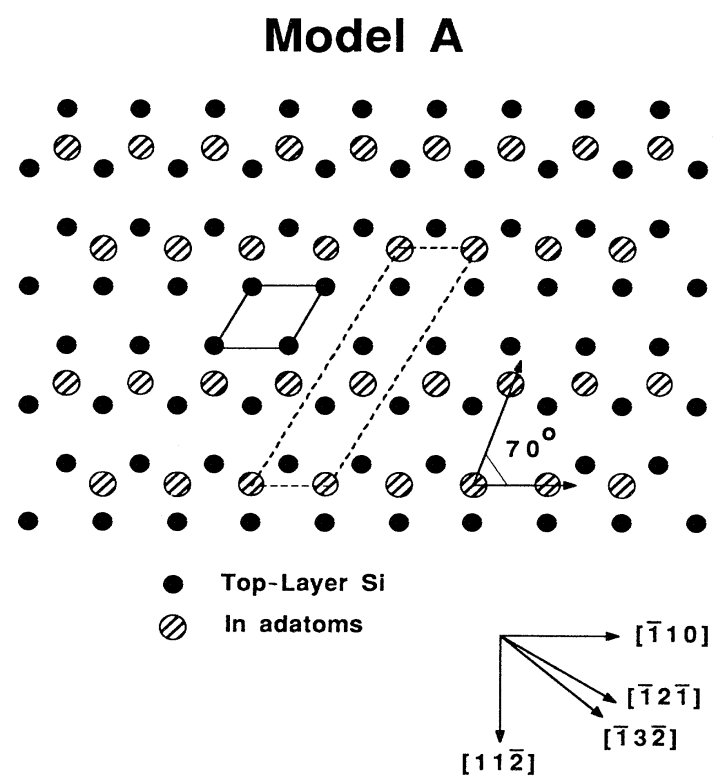

FIG. 1. Schematic plan view of model A for the Si(111)$(4 \times 1)$ In surface. The In adatoms sit in both $T_{4}$ and $H_{3}$ sites. Coverage is $\frac{1}{2}$ ML. The $1 \times 1$ and $4 \times 1$ unit cells are outlined.

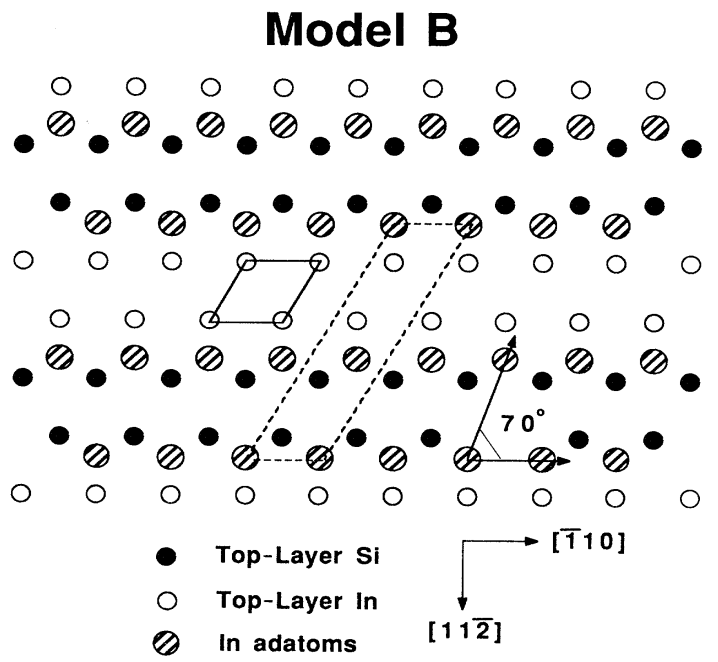

FIG. 2. Schematic plan view of model $B$ for the Si(111)$(4 \times 1)$ In surface. The In adatoms sit in both $T_{4}$ and $H_{3}$ sites. In atoms are also substituted into the first $\mathrm{Si}$ layer. Coverage is $1 \mathrm{ML}$. The $1 \times 1$ and $4 \times 1$ unit cells are outlined.

and 4. In model $\mathrm{C}$ (Fig. 3 ) the total In coverage is again $\frac{1}{2}$ ML while model D (Fig. 4) contains an additional $\frac{1}{2}$ ML of In in substitutional sites, yielding a total coverage of $1 \mathrm{ML}$.

The last model simulated is shown in Fig. 5. It is termed model $\mathrm{E}$ and contains $\frac{1}{2} \mathrm{ML}$ of In adatoms sitting in equivalent $\mathrm{H}_{3}$ sites. This model was simulated in our previous work. ${ }^{6}$

The adatom arrangement for models $\mathrm{A}$ and $\mathrm{B}$ more closely resembles the filled-state STM images, ${ }^{5}$ since each pair of adjacent atoms in the double row makes an angle of $70^{\circ}$ with the double-row axis. For models C, D, and E, however, the corresponding angle is $60^{\circ}$, as shown in

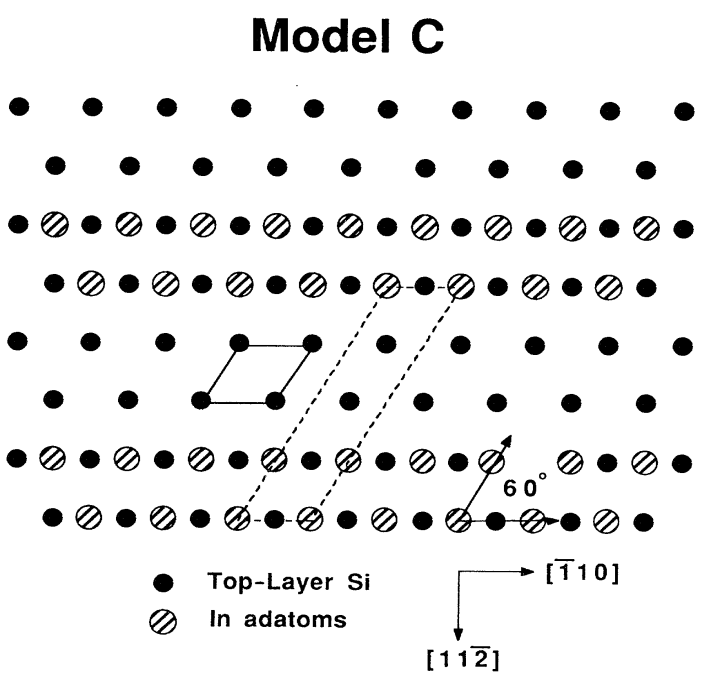

FIG. 3. Schematic plan view of model $\mathrm{C}$ for the Si(111)$(4 \times 1)$ In surface. The In adatoms sit in bridge sites. Coverage is $\frac{1}{2}$ ML. The $1 \times 1$ and $4 \times 1$ unit cells are outlined. 


\section{Model D}

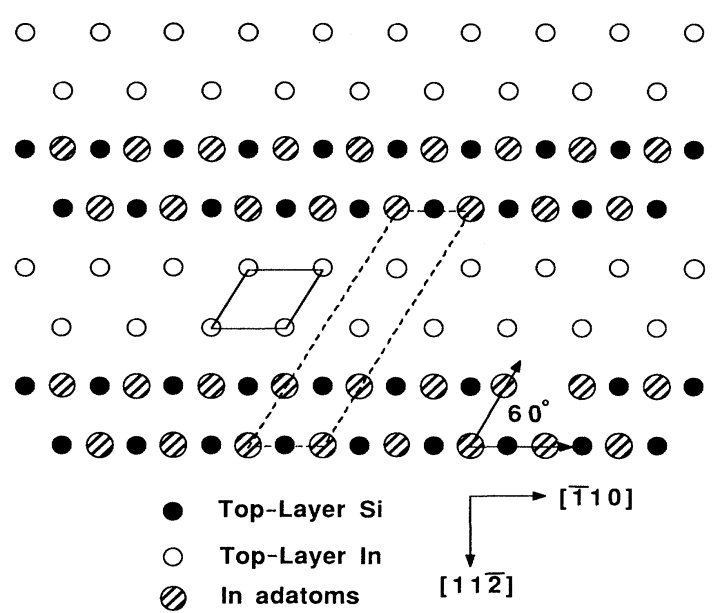

FIG. 4. Schematic plan view of model D for the Si(111)$(4 \times 1)$ In surface. The In adatoms sit in bridge sites. In atoms are also substituted into the first $\mathrm{Si}$ layer. Coverage is $1 \mathrm{ML}$. The $1 \times 1$ and $4 \times 1$ unit cells are outlined.

Figs. 3, 4, and 5, respectively.

Several missing top-layer models were also simulated during this study. However, they are not considered in the present paper since they do not provide better agreement despite their more complex reconstruction.

Polar-angle scans for $\mathrm{He}^{+}$ions scattered from both In and $\mathrm{Si}$ were taken for several incident azimuths. Since three equivalent orientations of the reconstruction exist, some azimuths may be equivalent. If the In atoms are positioned symmetrically with respect to the $1 \times 1$ surface mesh, then the number of unique directions to ion scattering is reduced. The $[\overline{1} 2 \overline{1}]\left(30^{\circ}\right.$ from $\left.[\overline{1} 10]\right)$ az-

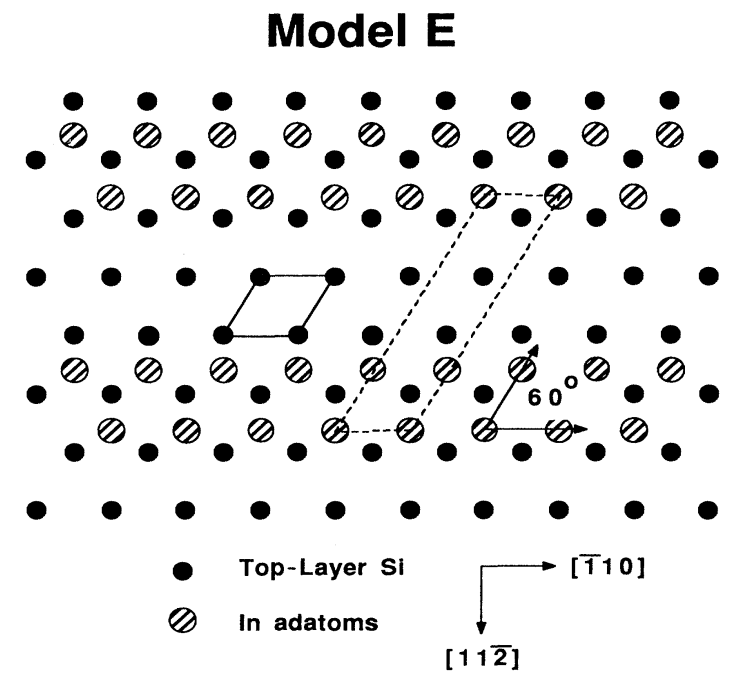

FIG. 5. Schematic plan view of model $\mathrm{E}$ for the $\mathrm{Si}(111)$ (4×1)In surface. The In adatoms all sit in $\mathrm{H}_{3}$ sites, and there is no substitutional In. Coverage is $\frac{1}{2}$ ML. The $1 \times 1$ and $4 \times 1$ unit cells are outlined. imuth bisects one orientation of the reconstruction. Due to reflection symmetry across this line an azimuth $+\theta$ from $[\overline{1} 2 \overline{1}]$ would then yield identical results to scattering as the azimuth $-\theta$ from $[\overline{1} 2 \overline{1}]$. If this is true, then scans along [110] (for example) should be identical to those along [011]. This was indeed observed. Since the $[11 \overline{2}]$ azimuth bisects another orientation of the reconstruction, the azimuths $[12 \overline{3}]$ and $[21 \overline{3}]$ should also yield the same results. This was also seen to be the case. Scattering data were taken for the azimuths $[\overline{1} 10],[\overline{1} 2 \overline{1}]$, $[\overline{1} 3 \overline{1}],[\overline{1} 3 \overline{2}],[\overline{1} 5 \overline{4}],[14 \overline{5}],[12 \overline{3}]$, and $[11 \overline{2}]$. All data support our final conclusions, but not all of them are presented in this paper.

The simulated polar-angle scans for each model were generated based upon calculations of two-atom-hitting probabilities. ${ }^{9}$ Both shadowing and blocking effects effects were simulated. The blocking effect made a notable contribution for a few azimuths for several models, especially for the $\mathrm{Si}$ simulations. The neutralization probability for the scattering $\mathrm{He}^{+}$ions was modeled after the model proposed by Engelmann, Taglauer, and Jackson (ETJ), ${ }^{10}$ which takes into account local interactions. In the ETJ model, three parameters are employed: the transition rate $A$, the inverse screening length $a^{-1}$, and the effective radius for local interactions $r$. The fitting parameters did not vary more than $10 \%$ from azimuth to azimuth.

Figure 6 shows the polar-angle scan for backscattering from In along the $[\overline{1} 10]$ direction. The experimental data contain a shoulder at $8^{\circ}$ and a peak at $20^{\circ}$. The simulations for each model are also shown in Fig. 6, the simulations being identical for models $\mathrm{C}, \mathrm{D}$, and $\mathrm{E}$. The neutralization parameters for the simulations are given in

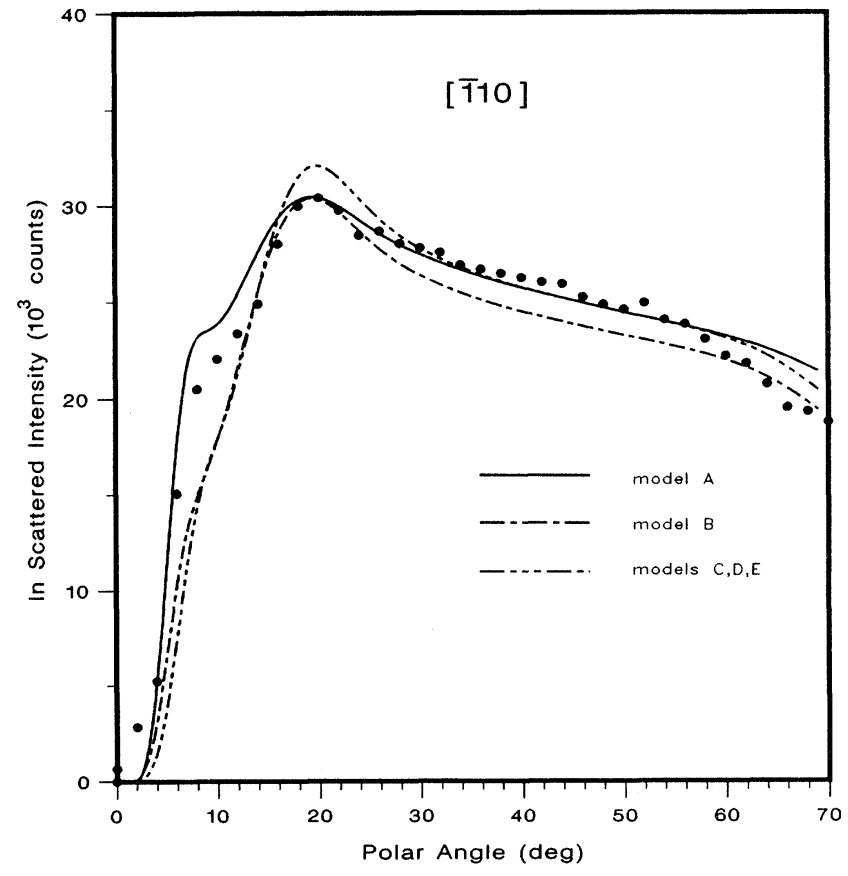

FIG. 6. ICISS polar-angle scan for $2-\mathrm{keV} \mathrm{He}^{+}$ions backscattered from In atoms along the [110] azimuth. Computer simulations for each model are indicated. 
TABLE I. Neutralization parameters used in simulation of polar-angle scans for models A-E. $A$ is a transition rate, $a$ is an inverse screening length, and $r$ is an effective radius for local interactions.

\begin{tabular}{ccccc}
\hline \hline & & & \multicolumn{2}{c}{$r(\AA)$} \\
Model & $A\left(\mathrm{fs}^{-1}\right)$ & $a\left(\AA^{-1}\right)$ & In & $\mathrm{Si}$ \\
\hline A & 1.0 & 0.95 & 1.2 & 0.9 \\
B & 0.9 & 1.1 & 1.1 & 0.85 \\
C & 1.0 & 0.95 & 1.1 & 0.85 \\
D & 0.95 & 1.1 & 1.1 & 0.9 \\
E & 1.0 & 1.0 & 1.2 & 0.9 \\
\hline \hline
\end{tabular}

Table I. It is interesting to note that these adjustable parameters do not vary appreciably from one model to another to give best fits. The peak at $8^{\circ}$ is due to In-In shadowing in the two other equivalent phases not running along [ $\overline{1} 10]$. The simulations for this feature combine In-In shadowing for linear displacements of 9.96, 11.52 , and $15.36 \AA$. The more prominent peak at $20^{\circ}$ results from In-In shadowing for a linear displacement of $3.84 \AA$. This feature is dominant in all the models simulated, and arises from the row geometry. All five models show good agreement with experiment. Model A, however, has a more pronounced feature at $8^{\circ}$ which more closely fits the experimental data. This is due to the lack of a lower substitutional layer of In, which contributes more intensity to the feature at $20^{\circ}$. Since only In-In shadowing is required to simulate the data, atoms at the topmost layer must be In adatoms.

The [110] azimuth polar-angle scan for backscattering from $\mathrm{Si}$ is shown in Fig. 7. The shoulder at $18^{\circ}$ is due to

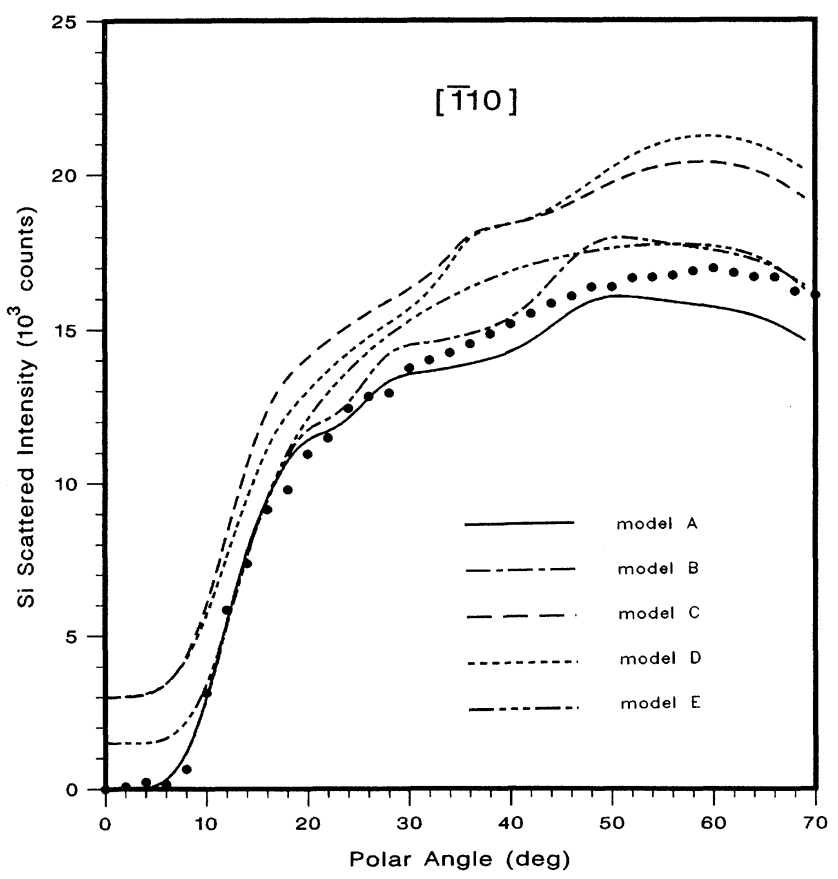

FIG. 7. ICISS polar-angle scan for $2-\mathrm{keV} \mathrm{He}^{+}$ions backscattered from $\mathrm{Si}$ atoms along the $[\overline{1} 10]$ azimuth. Computer simulations for each model are indicated.
Si-Si shadowing for a spacing of $3.84 \AA$. The experimental data also show small features at higher angles $\left(30^{\circ}\right.$ and $62^{\circ}$ ), but these are not entirely distinct. All models are shown and models C, D, and E are displaced by constant amounts to allow clear comparison of the features. Models $\mathbf{A}$ and $\mathbf{B}$ show some features at higher angles which are not clearly supported by the data. Model B, with less $\mathrm{Si}$ in the first $\mathrm{Si}$ layer, has more pronounced peaks. These result from the In adatom in the $T_{4}$ site shadowing the second Si layer atoms at spacings of $3.84,7.68$, and 11.52 $\AA$. Models $\mathrm{C}$ and $\mathrm{D}$ also have small features at $35^{\circ}$ due to second Si layer atoms shadowed by In adatoms. Model $\mathrm{C}$, which does not have substitutional In, has smaller features at these higher angles. Model E shows no higher-angle features; all the In adatoms are in $\mathrm{H}_{3}$ sites and do not shadow any $\mathrm{Si}$ atoms along this azimuth. The only dominant feature is that at $18^{\circ}$. The models with substitutional In, i.e., $\mathrm{B}$ and $\mathrm{D}$ show poorer agreement with the experimental data.

Scattering from In along the $[11 \overline{2}]$ and $[\overline{1} 2 \overline{1}]\left(30^{\circ}\right.$ from [ 110$]$ towards [ $11 \overline{2}]$ ) azimuths is shown in Figs. 8 and 9, respectively. The experimental scans show a shoulder at $14^{\circ}$ and no other features. Furthermore, the scans in both these azimuths appear very similar. If each In atom is shadowed only by other In atoms, then the symmetry of the rows to ion scattering (twofold) and the presence of three equivalent orientations will cause these two azimuths to yield the same results. The simulations for all the models are indicated. Models $\mathrm{C}$ and $\mathrm{E}$ give identical results since their In registrations are the same. Along either azimuth, it is clear that models $B$ and $D$ provide poor agreement with the experimental data. The pres-

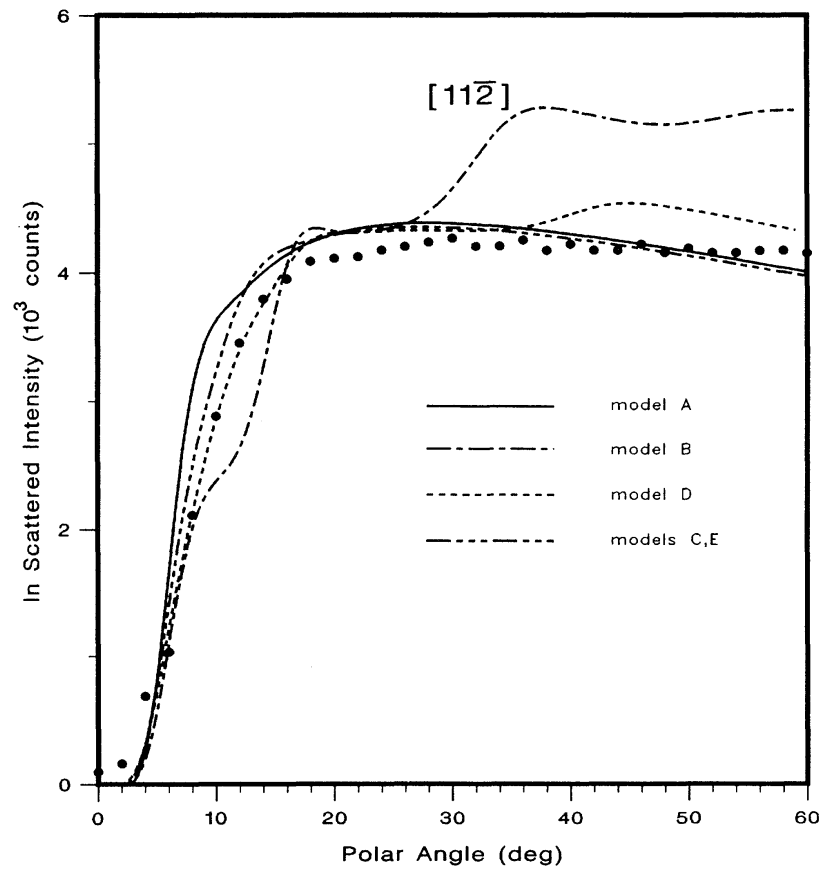

FIG. 8. ICISS polar-angle scan for $2-\mathrm{keV} \mathrm{He}^{+}$ions backscattered from In atoms along the $[11 \overline{2}]$ azimuth. Computer simulations for each model are indicated. 


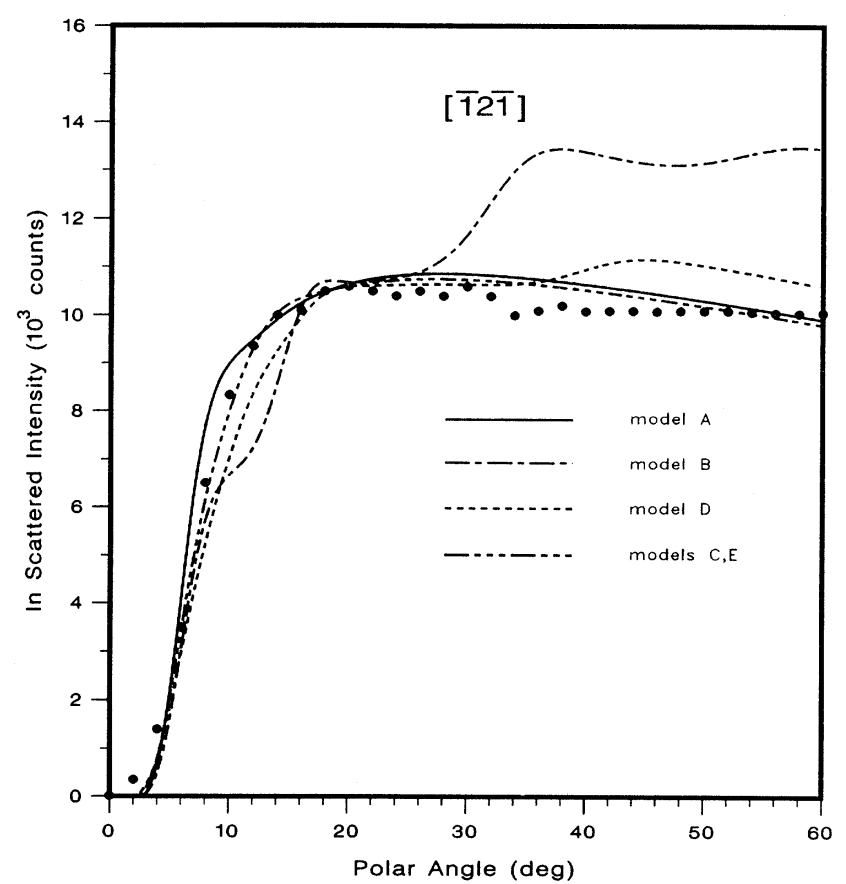

FIG. 9. ICISS polar-angle scan for $2-\mathrm{keV} \mathrm{He}^{+}$ions backscattered from In atoms along the $[\overline{1} 2 \overline{1}]$ azimuth. Computer simulations for each model are indicated.

ence of substitutional In atoms in these models yields higher-angle peaks which do not appear in the experimental scans. Models A, C, and E all provide reasonable agreement with experiment. The shoulder at $14^{\circ}$ results from the mixing of features for In-In shadowing for linear spacings of $11.06,13.3$, and $15.5 \AA$.

Scattering from Si along the [112] azimuth is shown in Fig. 10. The experimental data shows a small shoulder at $10^{\circ}$, a dominant peak at $30^{\circ}$, and a smaller peak at $60^{\circ}$. The shoulder at $10^{\circ}$ is due to the shadowing of first layer $\mathrm{Si}$ atoms by $\mathrm{Si}$ atoms $6.64 \AA$ away on the same layer. The increase in the scattering intensity with polar angle, however, is quickly blocked out by the In adatoms. Models A, C, and D show small simulated features at $10^{\circ}$ but their intensities are considerably smaller than the experimental intensities. The difference in intensities is probably a result of the uncertainty in estimating the neutralization for this blocking effect which gives rise to an underestimation of the $10^{\circ}$ shoulder. Model A provides the closest overall agreement with experiment, but models $\mathrm{C}$ and $\mathrm{D}$ show large discrepancies. Models $\mathrm{C}$ and $\mathrm{D}$ do not give a peak at $60^{\circ}$ since the shadowing conditions change for two of the three $4 \times 1$ orientations along this azimuth. The In adatoms in the bridge sites, for the orientations along [ $10 \overline{1}]$ and $[0 \overline{1} 1]$, are laterally displaced from the trajectory plane through all shadowed $\mathrm{Si}$ atoms. This displacement is too large for the adatoms to shadow the $\mathrm{Si}$ atoms. The scattering at $30^{\circ}$ results from atoms in the second $\mathrm{Si}$ layer which are shadowed by $\mathrm{Si}$ in the first layer with a linear displacement of $4.44 \AA$ and a vertical displacement of $0.77 \AA$. The peak at $60^{\circ}$ for models $\mathrm{A}, \mathrm{B}$, and $\mathrm{E}$ is a result of $\mathrm{In}-\mathrm{Si}$ shadowing from the second $\mathrm{Si}$

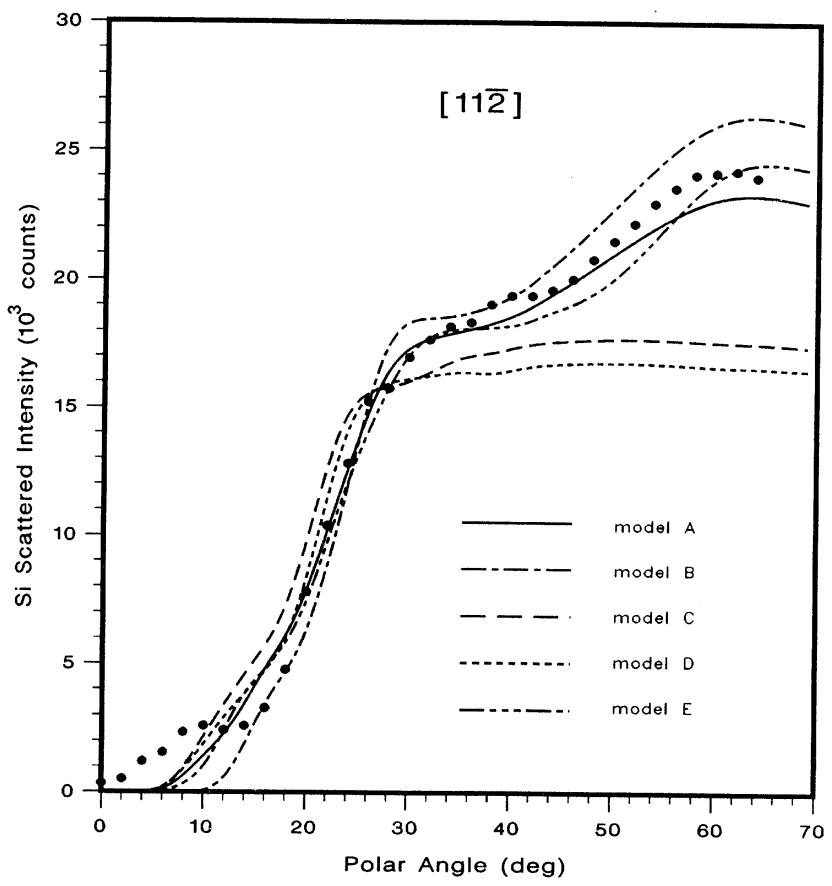

FIG. 10. ICISS polar-angle scan for $2-\mathrm{keV} \mathrm{He}^{+}$ions back-

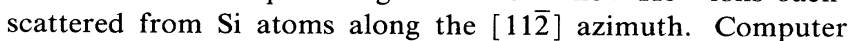
simulations for each model are indicated.

layer for linear spacing of $2.22 \AA$ and a vertical spacing of $\sim 2 \AA$.

The scattering from $\mathrm{Si}$ along the $[\overline{1} 2 \overline{1}]$ azimuth is shown in Fig. 11. There is a shoulder at $18^{\circ}$ and a large broad peak at $50^{\circ}$ in the experimental scan. All the models show general agreement with the data, but model $\mathrm{C}$ provides too much intensity at $10^{\circ}$. This shoulder results from a combination of several shadowing conditions with the dominant contribution coming from Si-Si shadowing for a linear spacing of $6.64 \AA$. The cause of the peak at $50^{\circ}$ is both $\mathrm{In}-\mathrm{Si}$ and $\mathrm{Si}-\mathrm{Si}$ shadowing for a linear spacing of $2.22 \AA$ and a vertical spacing of $0.77 \AA$. There are also other small contributions which add to the broadening of this peak.

Figure 12 shows the results for polar-angle scans for scattering from Si along the $[\overline{1} 3 \overline{2}]$ azimuth $\left(\sim 40^{\circ}\right.$ from [110] towards [112]). Only models $A$ and $E$ are shown in this plot. The other models are left out because their fits are far from satisfactory. The experimental data show a broad shoulder at $15^{\circ}$ and a peak at $42^{\circ}$. Model A is in closer agreement with the experimental data while model $\mathrm{E}$ shows more pronounced features at $10^{\circ}$ and $20^{\circ}$ which do not resemble the data. The peak at $15^{\circ}$ results from shadowing of first Si layer atoms by In atoms with a linear displacement of $10.15 \AA$ and no vertical displacement. Several other shadowing situations with similar spacings also exist for this azimuth. Indium shadowing $\mathrm{Si}$ atoms in the second $\mathrm{Si}$ layer with a linear displacement of only $2.22 \AA$ yield the peak at $42^{\circ}$.

After comparing the experimental polar scans with the computer-simulated scans along many azimuths, it becomes clear that the models with substitutional In and 1- 


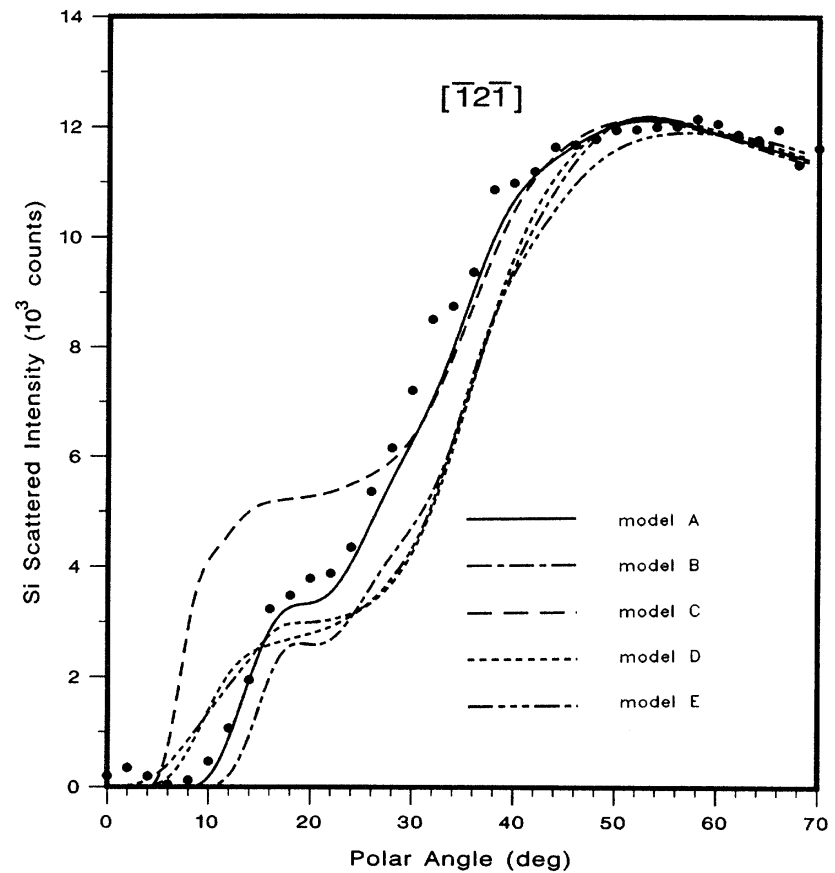

FIG. 11. ICISS polar-angle scan for $2-\mathrm{keV} \mathrm{He}^{+}$ions backscattered from $\mathrm{Si}$ atoms along the $[\overline{1} 2 \overline{1}]$ azimuth. Computer simulations for each model are indicated.

ML coverage, i.e., models $\mathbf{B}$ and $\mathbf{D}$, do not agree well with the experimental results. In particular, the [ $[\overline{1} 2 \overline{1}]$ and $[11 \overline{2}]$ In scans show no higher-angle features which would result from substitutional In atoms in the first $\mathrm{Si}$ layer. Of the remaining structures, model A supplies the best overall agreement, thus indicating that the $4 \times 1$ geometry consists of In adatoms occupying both $\mathrm{H}_{3}$ and $T_{4}$ sites with $\frac{1}{2}$-ML coverage. The bridge-site models $\mathrm{C}$

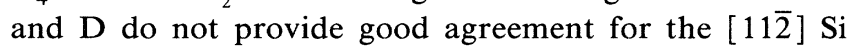
scan.

These results are not unique in the sense that other arrangements of In atoms possibly more complex than the models considered here could provide agreement with our experimental data. It is, however, abundantly clear from the In scans that In atoms reside on the top layer and are not shadowed by $\mathrm{Si}$ atoms.

The best fit to the experimental data for model A yields a vertical displacement of the In adatoms above the first Si layer of $1.15 \pm 0.15 \AA$. This produces an In-Si bond length of $2.5 \pm 0.1 \AA$.

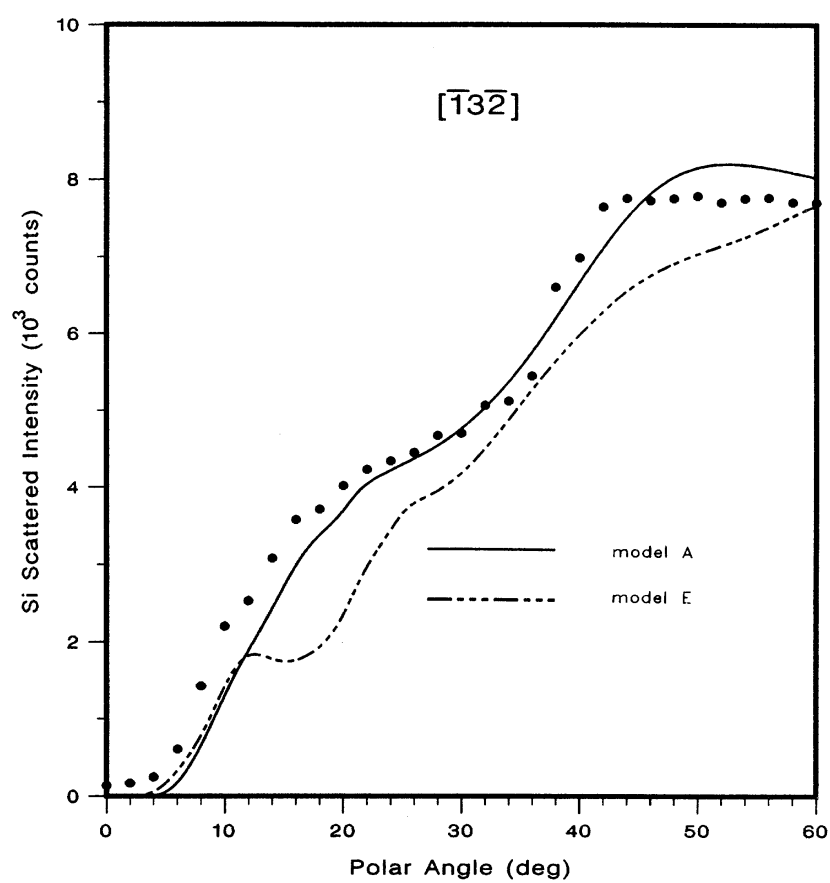

FIG. 12. ICISS polar-angle scan for $2-\mathrm{keV} \mathrm{He}^{+}$ions backscattered from $\mathrm{Si}$ atoms along the [132 $]$ azimuth. Computer simulations for models $\mathrm{A}$ and $\mathrm{E}$ are indicated.

\section{CONCLUSIONS}

The ICISS experiments show that the Si(111)-(4×1) In surface consists of a $\frac{1}{2}-\mathrm{ML}$ In coverage of the surface with the In atoms occupying the $\mathrm{H}_{3}$ and $T_{4}$ sites, consistent with model A proposed in the present work. The In adatoms reside above the first layer of $\mathrm{Si}$ with a vertical displacement of $1.15 \pm 0.15 \AA$ between the In and $\mathrm{Si}$ atoms. Our results are inconsistent with models of the $4 \times 1$ surface showing $1-$ ML coverage and substitutional In atoms in addition to In adatoms on the top-most layer.

\section{ACKNOWLEDGMENTS}

We thank J. Nogami and J. E. Northrup for many helpful discussions. This work was supported by the National Science Foundation under Grant No. DMR8718101 .
${ }^{1}$ M. Kawaji, S. Baba, and A. Kinbara, Appl. Phys. Lett. 34, 748 (1979).

2J. J. Lander and J. Morrison, Surf. Sci. 2, 553 (1964).

${ }^{3}$ J. M. Nicholls, P. Mårtensson, G. V. Hansson, and J. E. Northrup, Phys. Rev. B 32, 1333 (1985).

${ }^{4}$ J. Nogami, S. I. Park, and C. F. Quate, J. Vac. Sci. Technol. B 6, 1479 (1988).

${ }^{5}$ S. I. Park, J. Nogami, and C. F. Quate, J. Microsc. 152, 727 (1988).
${ }^{6}$ D. M. Cornelison, C. S. Chang, and I. S. T. Tsong, J. Vac. Sci. Technol. A 8, 3443 (1990).

${ }^{7}$ T. L. Porter, C. S. Chang, U. Knipping, and I. S. T. Tsong, Phys. Rev. B 36, 9150 (1987).

${ }^{8}$ S. I. Park, J. Nogami, and C. F. Quate (private communication).

${ }^{9}$ R. M. Tromp and J. F. van der Veen, Surf. Sci. 133, 159 (1983).

${ }^{10} \mathrm{G}$. Engelmann, E. Taglauer, and D. P. Jackson, Nucl. Instrum. Methods B 13, 240 (1986). 